Mathematical Modelling AND ANALysis

Volume 17 Number 2, April 2012, 227-244

http://dx.doi.org/10.3846/13926292.2012.662177

(c) Vilnius Gediminas Technical University, 2012
Publisher: Taylor\&Francis and VGTU

http://www.tandfonline.com/TMMA

Print ISSN: 1392-6292

Online ISSN: 1648-3510

\title{
Finite-Difference Analysis for the Linear Thermoporoelasticity Problem and Its Numerical Resolution by Multigrid Methods*
}

\author{
Natalia Boal ${ }^{a}$, Francisco José Gaspar ${ }^{a}$, \\ Francisco Lisbona $^{a}$ and Petr Vabishchevich ${ }^{b}$ \\ ${ }^{a}$ University of Zaragoza \\ Pedro Cerbuna 12, 50009 Zaragoza, Spain \\ ${ }^{b}$ Keldysh Institute of Applied Mathematics \\ 4-A Miusskaya Square, 125047 Moscow, Russia \\ E-mail: nboal@unizar.es \\ E-mail(corresp.): fjgaspar@unizar.es \\ E-mail: lisbona@unizar.es; vabishchevich@gmail.com
}

Received October 18, 2011; revised January 13, 2012; published online April 1, 2012

\begin{abstract}
This paper deals with the numerical solution of a two-dimensional thermoporoelasticity problem using a finite-difference scheme. Two issues are discussed: stability and convergence in discrete energy norms of the finite-difference scheme are proved, and secondly, a distributive smoother is examined in order to find a robust and efficient multigrid solver for the corresponding system of equations. Numerical experiments confirm the convergence properties of the proposed scheme, as well as fast multigrid convergence.
\end{abstract}

Keywords: finite-difference scheme, multigrid, finite-difference method.

AMS Subject Classification: 65N55; 65M12; 74F10; 74S10.

\section{Introduction}

The thermomechanical behavior of fluid saturated porous media is important in a number of diverse areas such as the extraction of geothermal energy, the design of nuclear waste storage sites and the enhanced oil recovery by hot fluid injection. Under common circumstances these problems involve strong coupling between heat transfer, motion of intersticial pore fluid, and deformation of the porous matrix.

\footnotetext{
* This research has been partially supported by FEDER/MCYT Projects MTM2010-16917 and the DGA (Grupo consolidado PDIE).
} 
The one-dimensional theory of isothermal consolidation was first formulated by Terzaghi [18]. The Terzaghi's theory was extended to a general 3D consolidation theory by Biot $[2,3]$, and a generalized formulation to the non-isothermal case was given by McTigue [14]. The main mechanisms of heat transfer into a geological formation are the heat conduction and the heat convection. The heat conduction is governed by Fourier's law while the heat convection depends on the rate of fluid flow. In this work, low permeable formations, such as granitic rock masses, are considered, where the rate of fluid flow is so slow that heat convection is negligible [14].

Thermoporoelasticity involves a coupling among three fields, displacement field, $\mathbf{u}(\mathbf{x}, t)$, pore pressure field, $p(\mathbf{x}, t)$, for fluid flow, and temperature field, $\theta(\mathbf{x}, t)$, for heat transfer. Neglecting convection, and nonlinear terms, the governing equations are then given by the system of equations

$$
\begin{gathered}
-\mu \Delta \mathbf{u}-(\lambda+\mu) \operatorname{grad} \operatorname{div} \mathbf{u}+\alpha_{p} \operatorname{grad} p+\alpha_{\theta} \operatorname{grad} \theta=\mathbf{g}, \\
\frac{1}{M} \frac{\partial p}{\partial t}-3 \beta_{M}^{\prime} \frac{\partial \theta}{\partial t}+\alpha_{p} \frac{\partial}{\partial t}(\operatorname{div} \mathbf{u})-k_{p} \Delta p=f^{p} \\
-3 \beta_{M}^{\prime} \frac{\partial p}{\partial t}+c_{M} \frac{\partial \theta}{\partial t}+\alpha_{\theta} \frac{\partial}{\partial t}(\operatorname{div} \mathbf{u})-k_{\theta} \Delta \theta=f^{\theta}
\end{gathered}
$$

where $\lambda$ and $\mu$ are the Lamé coefficients, $k_{p}$ and $k_{\theta}$ are the hydraulic and thermal conductivity respectively, $\alpha_{p}$ is the Biot-Willis constant, $\alpha_{\theta}$ is the thermal expansion coefficient of the solid phase, $M$ is the Biot modulus, $\beta_{M}^{\prime}$ is the equivalent thermal expansion coefficient, and $c_{M}$ is the equivalent volumetric heat capacity of the porous medium.

Appropriate initial and boundary conditions have to be added for the wellposedness of the mathematical model (1.1)-(1.3). For simplicity in the subsequent analysis, we assume here that $\partial \Omega$ is rigid (zero displacements), permeable (free drainage) and a null temperature is prescribed, so that we have homogeneous Dirichlet boundary conditions

$$
\mathbf{u}(\mathbf{x}, t)=\mathbf{0}, \quad p(\mathbf{x}, t)=0, \quad \theta(\mathbf{x}, t)=0, \quad \mathbf{x} \in \Omega, t>0 .
$$

The initial conditions are

$$
\mathbf{u}(\mathbf{x}, 0)=\mathbf{u}_{0}(\mathbf{x}), \quad p(\mathbf{x}, 0)=p_{0}(\mathbf{x}), \quad \theta(\mathbf{x}, 0)=\theta_{0}(\mathbf{x}), \quad \mathbf{x} \in \Omega .
$$

A detailed establishment of the governing equations for thermoporomechanics problems can be found in $[8,13]$.

In general, the solution of complex poromechanics problems is usually approximated by finite elements, see for instance the monograph by Lewis and Schrefler [13]. Problems where the solution is smooth are satisfactorily solved by standard finite element discretizations. Nevertheless, when strong pressure gradients appear, these methods are unstable in the sense that strong nonphysical oscillations appear in the approximation of the pressure field on each time level. It is well known that this phenomenon appears when materials have low permeability and a small time step is used. These nonphysical oscillations may be removed by a severer space mesh refinement, which is not practical. These oscillations can be minimized (but not completely alleviated without reducing the mesh size in space) if stable finite element methods are used. As for Stokes 
problems, approximation spaces for the vector and the scalar fields, satisfying the LBB stability condition [7], can be used. This approach has been analyzed, for example in [15] for the classical quasistatic Biot's model. Nevertheless, these methods still present small oscillations in pressure approximation when very sharp boundary layers occur.

Naturally, as for finite elements, standard finite-difference schemes may suffer the same unstable behaviour in pressure approximation. In [11], a reason for this instability for one-dimensional consolidation problems has been identified, and that leads to use staggered grid discretizations for poroelasticity problems [12]. Finite-difference methods on staggered grids lead to stable discretizations that mimic the continuous problem very well, so that the main properties of the continuous problem are preserved in the discrete approach. Notice also that the use of staggered grids is the way to incorporate a discrete inf-sup condition in the finite-difference framework, see for example [17]. For other Biot's models, staggered grids have also been successfully applied, such as the secondary consolidation model [9], the double porosity model [5] and the fully dynamic problem [4]. For these reasons, we apply this method to linear thermoporoelasticity problems in order to preclude the pressure and temperature oscillations. In Section 2, a spatial discretization on staggered grids, such that the main properties of the differential operators are preserved in the discrete level is proposed. In Section 3, a priori estimates in discrete energy norm are obtained for displacements, pressure and temperature, and convergence results are given.

The design of efficient smoothers in multigrid for the iterative solution of systems of partial differential equations, often requires special attention. The relaxation method should smooth the error for all unknowns in the equations of the system. Smoothing difficulties are met if one of the operators on the system's main diagonal equals zero, or is very close to zero (i.e. with extremely small parameters in front of derivatives). A possibility to overcome this difficulty is to consider distributive smoothers. In distributive smoothing the original system of equations is transformed by post-conditioning in order to achieve favorable properties, such as a decoupling of the equations and/or possibilities for point-wise smoothing. The research underlying these relaxation methods for incompressible flow problems dates basically back to the late 1970's [6]. A distributive, decoupled relaxation method for poroelasticity has been recently introduced in $[10,20]$. The development of such smoothers for the thermoporoelaticity problem is pursued in Section 4. In Section 5 numerical experiments confirming the theoretical results of the difference scheme are shown. Besides, numerical multigrid results are presented, showing its robustness w.r.t. the problem parameters, as Lamé coefficients, conductivities, and time step and grid size.

\section{A Difference Scheme}

\subsection{Grids and grid-operators}

For simplicity in notation, we consider the problem on the unit square and introduce uniform grids with the same mesh size in each direction. Let $\mathcal{G}_{n}$ be 
the set of nodes of an uniform partition of the interval $[0,1]$

$$
\mathcal{G}_{n}=\{k h \mid k=0, \ldots, N\}
$$

where $h=1 / N$, with $N \in \mathbb{N}$. Related to this partition, let $\mathcal{G}_{c}$ be the set of the center nodes

$$
\mathcal{G}_{c}=\left\{\left(k+\frac{1}{2}\right) h \mid k=-1 / 2,0, \ldots, N-1, N-1 / 2\right\} .
$$

Associated with the different unknowns of our problem, we introduce the meshes $\bar{\omega}_{u}$ and $\bar{\omega}_{v}$ to approximate the horizontal and vertical components of the displacement vector, and the mesh $\bar{\omega}_{p}$ to approximate the scalar fields, i.e., the pressure and the temperature

$$
\bar{\omega}_{p}=\mathcal{G}_{n} \times \mathcal{G}_{n}, \quad \bar{\omega}_{u}=\mathcal{G}_{c} \times \mathcal{G}_{n}, \quad \bar{\omega}_{v}=\mathcal{G}_{n} \times \mathcal{G}_{c} .
$$

Besides, let us denote $\omega_{p}, \omega_{u}, \omega_{v}$, the set of internal nodes and $\partial \omega_{p}, \partial \omega_{u}, \partial \omega_{v}$, the set of boundary nodes on $\bar{\omega}_{p}, \bar{\omega}_{u}, \bar{\omega}_{v}$, respectively.

Related to these meshes, we define the corresponding Hilbert spaces of grid-functions $H_{\bar{\omega}_{p}}, H_{\bar{\omega}_{u}}, H_{\bar{\omega}_{v}}$ and $\mathbf{H}_{\overline{\boldsymbol{\omega}}_{u}}=H_{\bar{\omega}_{u}} \times H_{\bar{\omega}_{v}}$ with usual $L_{2}$ inner products [16]. We also denote by $H_{\omega_{p}}, H_{\omega_{u}}, H_{\omega_{v}}$ and $\mathbf{H}_{\omega_{u}}=H_{\omega_{u}} \times H_{\omega_{v}}$ the subspaces of grid functions vanishing on the boundaries. For instance, for grid-functions defined on $H_{\omega_{p}}$, the inner product and norm are as follows

$$
(p, q)=\sum_{\mathbf{x} \in \omega_{p}} p(\mathbf{x}) q(\mathbf{x}) h^{2}, \quad\|p\|=(p, p)^{1 / 2} .
$$

For vector grid-functions defined on $\bar{\omega}_{p}$ vanishing on the boundaries, we also introduce the Hilbert spaces $\mathbf{H}_{\boldsymbol{\omega}_{p}}=H_{\omega_{p}} \times H_{\omega_{p}}$ with the inner product and norm given by

$$
(\mathbf{p}, \mathbf{q})=\left(p_{1}, q_{1}\right)+\left(p_{2}, q_{2}\right), \quad\|\mathbf{p}\|=(\mathbf{p}, \mathbf{p})^{1 / 2} .
$$

and $\mathbf{H}_{\bar{\omega}_{p}}=H_{\bar{\omega}_{p}} \times H_{\bar{\omega}_{p}}$ with the corresponding inner product and norm. Also, given an operator $T$ selfadjoint and positive on a Hilbert space $H$, we will use the inner product $(p, q)_{T}=(T p, q)$ and the corresponding norm $\|p\|_{T}$.

\subsection{Discretization}

Now, we introduce the suitable discrete operators used in the spatial discretization. To define the discrete operators involved in the equations of our problem (1.1)-(1.3), we take as basic (or support) operator the following discrete divergence operator $D: \mathbf{H}_{\overline{\boldsymbol{\omega}}_{u}} \rightarrow H_{\bar{\omega}_{p}}$ defined by $(D \mathbf{u})_{i, j}=\left(D^{x} u\right)_{i, j}+\left(D^{y} v\right)_{i, j}$, where

$$
\left(D^{x} u\right)_{i, j}=\frac{u_{i+1 / 2, j}-u_{i-1 / 2, j}}{h}, \quad\left(D^{y} v\right)_{i, j}=\frac{v_{i, j+1 / 2}-v_{i, j-1 / 2}}{h}
$$

for the internal points, and

$$
\begin{gathered}
\left(D^{x} u\right)_{0, j}=\frac{2}{h}\left(u_{1 / 2, j}-u_{0, j}\right), \quad\left(D^{x} u\right)_{N, j}=\frac{2}{h}\left(u_{N, j}-u_{N-1 / 2, j}\right), \quad j=0, \ldots N, \\
\left(D^{y} v\right)_{i, 0}=\frac{2}{h}\left(v_{i, 1 / 2}-v_{i, 0}\right), \quad\left(D^{y} v\right)_{i, N}=\frac{2}{h}\left(v_{i, N}-v_{i, N-1 / 2}\right), \quad i=0, \ldots N,
\end{gathered}
$$


on the boundary. The discrete gradient operator $G: H_{\bar{\omega}_{p}} \rightarrow \mathbf{H}_{\bar{\omega}_{u}}$, whose components are located on displacement points, is defined as the negative adjoint to operator $D$, i.e.,

$$
\left(G p_{h}, \mathbf{u}_{h}\right)=-\left(p_{h}, D \mathbf{u}_{h}\right), \quad \forall \mathbf{u}_{h} \in \mathbf{H}_{\overline{\boldsymbol{\omega}}_{u}}, p_{h} \in H_{\bar{\omega}_{p}} .
$$

Based on the discrete operators $D$ and $G$, we now consider operators $\widetilde{G}: \mathbf{H}_{\overline{\boldsymbol{\omega}}_{p}} \rightarrow$ $\mathbf{H}_{\overline{\boldsymbol{\omega}}_{u}}$ and $\widetilde{D}: \mathbf{H}_{\overline{\boldsymbol{\omega}}_{u}} \rightarrow \mathbf{H}_{\overline{\boldsymbol{\omega}}_{p}}$ defined by $\widetilde{G} \mathbf{p}_{h}=\widetilde{G}\left(p_{h}, \theta_{h}\right)=\alpha_{p} G p_{h}+\alpha_{\theta} G \theta_{h}$ and $\widetilde{D} \mathbf{u}_{h}=\left(\alpha_{p} D \mathbf{u}_{h}, \alpha_{\theta} D \mathbf{u}_{h}\right)^{t}$ respectively. From relation (2.2), it is immediate that operators $\widetilde{G}$ and $\widetilde{D}$ satisfy a similar property, i.e.,

$$
\left(\widetilde{G} \mathbf{p}_{h}, \mathbf{u}_{h}\right)=-\left(\mathbf{p}_{h}, \widetilde{D} \mathbf{u}_{h}\right), \quad \forall \mathbf{u}_{h} \in \mathbf{H}_{\bar{\omega}_{u}}, \mathbf{p}_{h} \in \mathbf{H}_{\overline{\boldsymbol{\omega}}_{p}} .
$$

To discretize the elasticity operator, we define the operator $A: \mathbf{H}_{\boldsymbol{\omega}_{u}} \rightarrow \mathbf{H}_{\boldsymbol{\omega}_{u}}$ by

$$
A=-\mu \Delta_{h}^{\mathbf{u}}-(\lambda+\mu) G D,
$$

where $\Delta_{h}^{\mathbf{u}}=\operatorname{diag}\left(\Delta_{h}^{u}, \Delta_{h}^{v}\right)$, being $\Delta_{h}^{u}$ and $\Delta_{h}^{v}$ the five point Laplacian approximation formula on the grids $\omega_{u}$ and $\omega_{v}$, respectively. Operator $A$ is self-adjoint and positive definite on $\mathbf{H}_{\boldsymbol{\omega}_{u}}$, so $A \geq \delta_{\mathcal{A}} E$ where $\delta_{\mathcal{A}}>0$ is independent of $h$.

The semi-discrete approximations $\mathbf{u}_{h}(t)=\left(u_{h}(t), v_{h}(t)\right) \in \mathbf{H}_{\boldsymbol{\omega}_{u}}$ and $\mathbf{p}_{h}(t)=$ $\left(p_{h}(t), \theta_{h}(t)\right) \in \mathbf{H}_{\boldsymbol{\omega}_{p}}$ of the solution of continuous problem (1.1)-(1.3) are given as the solution of the difference-differential system:

$$
\begin{aligned}
A \mathbf{u}_{h}(t)+\widetilde{G} \mathbf{p}_{h}(t) & =\mathbf{g}_{h}(t), \\
\frac{\mathrm{d}}{\mathrm{d} t}\left(\widetilde{D} \mathbf{u}_{h}(t)+C \mathbf{p}_{h}(t)\right)+B \mathbf{p}_{h}(t) & =\mathbf{f}_{h}(t),
\end{aligned}
$$

for all $t \in(0, T]$, with initial conditions

$$
\mathbf{u}_{h}(0)=\mathbf{u}_{0}, \quad \mathbf{p}_{h}(0)=\left(p_{0}, \theta_{0}\right) .
$$

In equation (2.5) the right hand side is $\mathbf{f}_{h}(t)=\left(f_{h}^{p}(t), f_{h}^{\theta}(t)\right)$ and operators $C, B: \mathbf{H}_{\boldsymbol{\omega}_{p}} \rightarrow \mathbf{H}_{\boldsymbol{\omega}_{p}}$ given by

$$
C=\left(\begin{array}{cc}
1 / M & -3 \beta_{M}^{\prime} \\
-3 \beta_{M}^{\prime} & c_{M}
\end{array}\right), \quad B=\left(\begin{array}{cc}
-k_{p} \Delta_{h} & 0 \\
0 & -k_{\theta} \Delta_{h}
\end{array}\right),
$$

satisfy that $C=C^{*} \geq \delta_{C} E, \delta_{C}>0$, and $B=B^{*} \geq \delta_{B} E, \delta_{B}>0$, i.e. $C$ and $B$ are symmetric and positive definite operators in $\mathbf{H}_{\boldsymbol{\omega}_{p}}$. These properties of operator $C$ agree with the characteristics of many materials, as for example rocks (see, for example [1]). Here, $\Delta_{h}=D G$ is the usual five-point stencil approximation for the Laplace operator on $H_{\omega_{p}}$.

To obtain the fully discrete scheme, we apply a simple time discretization process to the problem (2.4)-(2.5). For simplicity, a uniform grid in $[0, T]$, with step $\tau>0$ is chosen. Let $\mathbf{p}_{h}^{m}=\mathbf{p}_{h}\left(t^{m}\right), \mathbf{u}_{h}^{m}=\mathbf{u}_{h}\left(t^{m}\right), t^{m}=m \tau$, $m=0,1, \ldots, M, M \tau=T$. In this way, system (2.4)-(2.5) is approximated by the following scheme

$$
\begin{aligned}
A \mathbf{u}_{h}^{m+1}+\widetilde{G} \mathbf{p}_{h}^{m+1} & =\mathbf{g}_{h}^{m+1}, \\
\widetilde{D} \frac{\mathbf{u}_{h}^{m+1}-\mathbf{u}_{h}^{m}}{\tau}+C \frac{\mathbf{p}_{h}^{m+1}-\mathbf{p}_{h}^{m}}{\tau}+B \mathbf{p}_{h}^{m+1} & =\mathbf{f}_{h}^{m+1},
\end{aligned}
$$


with initial conditions

$$
\mathbf{u}_{h}^{0}=\mathbf{u}_{0}, \quad \mathbf{p}_{h}^{0}=\left(p_{0}, \theta_{0}\right)
$$

\section{$3 \quad$ Stability Estimates and Convergence}

In this section we investigate the stability and convergence of difference scheme (2.7)-(2.8) in discrete energy norms with respect to the initial data and the right hand side.

Lemma 1. Let be $\mathbf{g}_{h}^{m+1}=\mathbf{0}, m=0, \ldots, M-1$, in (2.7), then the solution of problem (2.7)-(2.8) satisfies the a priori estimate

$$
\left\|\mathbf{u}_{h}^{m+1}\right\|_{A}^{2}+\left\|\mathbf{p}_{h}^{m+1}\right\|_{C}^{2} \leq\left\|\mathbf{u}_{h}^{m}\right\|_{A}^{2}+\left\|\mathbf{p}_{h}^{m}\right\|_{C}^{2}+\frac{\tau}{2}\left\|\mathbf{f}_{h}^{m+1}\right\|_{B^{-1}}^{2}
$$

for $m=0,1, \ldots, M-1$.

Proof. Multiplying scalarly (2.7) and (2.8) by $\frac{\mathbf{u}_{h}^{m+1}-\mathbf{u}_{h}^{m}}{\tau}$ and $\mathbf{p}_{h}^{m+1}$ respectively, adding the resulting equations, and using relation (2.3), we get for $m=0,1, \ldots, M-1$

$$
\left(A \mathbf{u}_{h}^{m+1}, \frac{\mathbf{u}_{h}^{m+1}-\mathbf{u}_{h}^{m}}{\tau}\right)+\left(C \frac{\mathbf{p}_{h}^{m+1}-\mathbf{p}_{h}^{m}}{\tau}, \mathbf{p}_{h}^{m+1}\right)+\left\|\mathbf{p}_{h}^{m+1}\right\|_{B}^{2}=\left(\mathbf{f}_{h}^{m+1}, \mathbf{p}_{h}^{m+1}\right) .
$$

Using the Cauchy-Schwarz inequality in the right-hand side,

$$
\left(\mathbf{f}_{h}^{m+1}, \mathbf{p}_{h}^{m+1}\right) \leq\left\|\mathbf{p}_{h}^{m+1}\right\|_{B}^{2}+\frac{1}{4}\left\|\mathbf{f}_{h}^{m+1}\right\|_{B^{-1}}^{2},
$$

and the identity

$$
\begin{aligned}
2 \tau\left(R \mathbf{y}_{h}^{m+1}, \frac{\mathbf{y}_{h}^{m+1}-\mathbf{y}_{h}^{m}}{\tau}\right)= & \left(R\left(\mathbf{y}_{h}^{m+1}+\mathbf{y}_{h}^{m}\right), \mathbf{y}_{h}^{m+1}-\mathbf{y}_{h}^{m}\right) \\
& +\tau^{2}\left(R \frac{\mathbf{y}_{h}^{m+1}-\mathbf{y}_{h}^{m}}{\tau}, \frac{\mathbf{y}_{h}^{m+1}-\mathbf{y}_{h}^{m}}{\tau}\right)
\end{aligned}
$$

which is valid for any symmetric and positive definite operator $R$, we have

$$
\frac{1}{2}\left(\left\|\mathbf{u}_{h}^{m+1}\right\|_{A}^{2}-\left\|\mathbf{u}_{h}^{m}\right\|_{A}^{2}\right)+\frac{1}{2}\left(\left\|\mathbf{p}_{h}^{m+1}\right\|_{C}^{2}-\left\|\mathbf{p}_{h}^{m}\right\|_{C}^{2}\right) \leq \frac{\tau}{4}\left\|\mathbf{f}_{h}^{m+1}\right\|_{B^{-1}}^{2},
$$

and estimate (3.1) follows.

Proposition 1. The solution of problem (2.7)-(2.8) satisfies the a priori estimate

$$
\begin{aligned}
\left\|\mathbf{u}_{h}^{m+1}\right\|_{A}^{2}+\left\|\mathbf{p}_{h}^{m+1}\right\|_{C}^{2} \leq & 2\left\|\mathbf{u}_{h}^{0}\right\|_{A}^{2}+2\left\|\mathbf{p}_{h}^{0}\right\|_{C}^{2}+2\left\|\mathbf{g}_{h}^{m+1}\right\|_{A^{-1}}^{2} \\
& +\tau \sum_{k=0}^{m}\left[\left\|\mathbf{f}_{h}^{k+1}\right\|_{B^{-1}}^{2}+K\left\|\frac{\mathbf{g}_{h}^{k+1}-\mathbf{g}_{h}^{k}}{\tau}\right\|_{A^{-1}}^{2}\right],
\end{aligned}
$$

for $m=0,1, \ldots, M-1$ and where $K$ is a constant independent of $h$ and $\tau$. 
Proof. Displacement vector $\mathbf{u}_{h}^{m+1}$, solution of problem (2.7)-(2.8), is split up in two parts, $\mathbf{u}_{h}^{m+1}=\overline{\mathbf{u}}_{h}^{m+1}+\overline{\overline{\mathbf{u}}}_{h}^{m+1}, m=0, \ldots M-1$, where $\overline{\mathbf{u}}_{h}^{m+1}$ is the solution of the problem

$$
A \overline{\mathbf{u}}_{h}^{m+1}=\mathbf{g}_{h}^{m+1}, \quad \overline{\mathbf{u}}_{h}^{m+1} \in \mathbf{H}_{\boldsymbol{\omega}_{u}},
$$

and $\overline{\overline{\mathbf{u}}}_{h}^{m+1}$ is the solution of the problem

$$
\begin{aligned}
A \overline{\overline{\mathbf{u}}}_{h}^{m+1}+\widetilde{G} \mathbf{p}_{h}^{m+1} & =\mathbf{0}, \\
\widetilde{D} \frac{\overline{\overline{\mathbf{u}}}_{h}^{m+1}-\overline{\overline{\mathbf{u}}}_{h}^{m}}{\tau}+C \frac{\mathbf{p}_{h}^{m+1}-\mathbf{p}_{h}^{m}}{\tau}+B \mathbf{p}_{h}^{m+1} & =\widetilde{\mathbf{f}}_{h}^{m+1},
\end{aligned}
$$

with

$$
\widetilde{\mathbf{f}}_{h}^{m+1}=\mathbf{f}_{h}^{m+1}-\widetilde{D} \frac{\overline{\mathbf{u}}_{h}^{m+1}-\overline{\mathbf{u}}_{h}^{m}}{\tau}, \quad \overline{\overline{\mathbf{u}}}_{h}^{0}=\mathbf{u}_{h}^{0}, \quad \text { and } \quad \overline{\mathbf{u}}_{h}^{0}=\mathbf{0} .
$$

For the solution of problem (3.3) we have $\left\|\overline{\mathbf{u}}_{h}^{m+1}\right\|_{A}=\left\|\mathbf{g}_{h}^{m+1}\right\|_{A^{-1}}$. Applying Lemma 1 to problem (3.4)-(3.5), we obtain the estimate

$$
\begin{aligned}
\left\|\overline{\overline{\mathbf{u}}}_{h}^{m+1}\right\|_{A}^{2}+\left\|\mathbf{p}_{h}^{m+1}\right\|_{C}^{2} \leq & \left\|\overline{\overline{\mathbf{u}}}_{h}^{m}\right\|_{A}^{2}+\left\|\mathbf{p}_{h}^{m}\right\|_{C}^{2}+\frac{\tau}{2}\left\|\mathbf{f}_{h}^{m+1}\right\|_{B^{-1}}^{2} \\
& +\frac{\tau\left(\alpha_{p}^{2}+\alpha_{\theta}^{2}\right)}{2 \delta_{B}(\lambda+\mu)}\left\|\frac{\mathbf{g}_{h}^{m+1}-\mathbf{g}_{h}^{m}}{\tau}\right\|_{A^{-1}}^{2}
\end{aligned}
$$

where we have used the following relation

$$
\begin{aligned}
(\lambda+\mu) \| & \widetilde{D}_{h} \frac{\overline{\mathbf{u}}_{h+1}^{m+1}-\overline{\mathbf{u}}_{h}^{m}}{\tau} \|_{B^{-1}}^{2} \leq \frac{(\lambda+\mu)}{\delta_{B}}\left(\widetilde{D} \frac{\overline{\mathbf{u}}_{h}^{m+1}-\overline{\mathbf{u}}_{h}^{m}}{\tau}, \widetilde{D}_{\frac{\mathbf{u}_{h}^{m+1}}{\tau}-\overline{\mathbf{u}}_{h}^{m}}\right) \\
& =-\frac{(\lambda+\mu)}{\delta_{B}}\left(\widetilde{G} \widetilde{D} \frac{\overline{\mathbf{u}}_{h}^{m+1}-\overline{\mathbf{u}}_{h}^{m}}{\tau}, \frac{\overline{\mathbf{u}}_{h}^{m+1}-\overline{\mathbf{u}}_{h}^{m}}{\tau}\right) \\
& \leq\left(\frac{\alpha_{p}^{2}+\alpha_{\theta}^{2}}{\delta_{B}}\right)\left\|\frac{\overline{\mathbf{u}}_{h}^{m+1}-\overline{\mathbf{u}}_{h}^{m}}{\tau}\right\|_{A}^{2}=\left(\frac{\alpha_{p}^{2}+\alpha_{\theta}^{2}}{\delta_{B}}\right)\left\|\frac{\mathbf{g}_{h}^{m+1}-\mathbf{g}_{h}^{m}}{\tau}\right\|_{A^{-1}}^{2}
\end{aligned}
$$

By recursion,

$$
\begin{aligned}
\left\|\overline{\overline{\mathbf{u}}}_{h}^{m+1}\right\|_{A}^{2}+\left\|\mathbf{p}_{h}^{m+1}\right\|_{C}^{2} \leq & \left\|\overline{\overline{\mathbf{u}}}_{h}^{0}\right\|_{A}^{2}+\left\|\mathbf{p}_{h}^{0}\right\|_{C}^{2} \\
& +\frac{\tau}{2} \sum_{k=0}^{m}\left[\left\|\mathbf{f}_{h}^{k+1}\right\|_{B^{-1}}^{2}+\frac{\left(\alpha_{p}^{2}+\alpha_{\theta}^{2}\right)}{(\lambda+\mu) \delta_{B}}\left\|\frac{\mathbf{g}_{h}^{k+1}-\mathbf{g}_{h}^{k}}{\tau}\right\|_{A^{-1}}^{2}\right] .
\end{aligned}
$$

Taking into account $\left\|\mathbf{u}_{h}^{m+1}\right\|_{A}^{2} \leq 2\left\|\overline{\mathbf{u}}_{h}^{m+1}\right\|_{A}^{2}+2\left\|\overline{\overline{\mathbf{u}}}_{h}^{m+1}\right\|_{A}^{2}$, estimate (3.2) is obtained.

Let us denote the error functions of the discrete solutions as follows,

$$
\begin{aligned}
& \delta \mathbf{u}_{h}^{m}(\mathbf{x})=\mathbf{u}_{h}^{m}(\mathbf{x})-\mathbf{u}\left(\mathbf{x}, t_{m}\right) \in \mathbf{H}_{\boldsymbol{\omega}_{u}}, \quad m=0, \ldots, M, \\
& \delta \mathbf{p}_{h}^{m}(\mathbf{x})=\left(p_{h}^{m}(\mathbf{x})-p\left(\mathbf{x}, t_{m}\right), \theta_{h}^{m}(\mathbf{x})-\theta\left(\mathbf{x}, t_{m}\right)\right) \in \mathbf{H}_{\boldsymbol{\omega}_{p}}, \quad m=0, \ldots, M .
\end{aligned}
$$


By substituting these functions into the finite-difference scheme (2.7)-(2.8), we get a discrete problem for the error functions:

$$
\begin{aligned}
A \delta \mathbf{u}_{h}^{m+1}+\widetilde{G} \delta \mathbf{p}_{h}^{m+1} & =\boldsymbol{\Psi}_{h}^{m+1}, \\
\widetilde{D} \frac{\delta \mathbf{u}_{h}^{m+1}-\delta \mathbf{u}_{h}^{m}}{\tau}+C \frac{\delta \mathbf{p}_{h}^{m+1}-\delta \mathbf{p}_{h}^{m}}{\tau}+B \delta \mathbf{p}_{h}^{m+1} & =\boldsymbol{\Phi}_{h}^{m+1},
\end{aligned}
$$

with initial conditions

$$
\delta \mathbf{u}_{h}^{0}=\mathbf{0}, \quad \delta \mathbf{p}_{h}^{0}=\mathbf{0},
$$

where functions $\boldsymbol{\Psi}_{h}^{m+1}$ and $\boldsymbol{\Phi}_{h}^{m+1}$ are the approximation errors. Let us assume regularity and compatibility conditions for initial and boundary conditions such that the exact solution satisfies $(\mathbf{u}(\mathbf{x}, t), p(\mathbf{x}, t), \theta(\mathbf{x}, t)) \in C_{2}^{6}(\bar{\Omega} \times[0, T])$. Then, Taylor expansion shows that $\boldsymbol{\Psi}_{h}^{m+1}(\mathbf{x})=\mathcal{O}\left(h^{2}\right)$ and $\left(\boldsymbol{\Psi}_{h}^{m+1}(\mathbf{x})-\boldsymbol{\Psi}_{h}^{m}(\mathbf{x})\right) / \tau=$ $\mathcal{O}\left(h^{2}\right)$ for $\mathbf{x} \in \omega_{\mathbf{u}}$, and $\mathbf{\Phi}_{h}^{m+1}(\mathbf{x})=\mathcal{O}\left(h^{2}+\tau\right)$ for $\mathbf{x} \in \omega_{\mathbf{p}}$. Noting that $\|\cdot\|_{A^{-1}} \leq$ $\left(1 / \delta_{A}\right)\|\cdot\|$, error estimates follow from the stability of the difference scheme, and as a consequence, the next convergence result is obtained.

Proposition 2. Assume that $\left(\mathbf{u}_{h}^{m+1}, \mathbf{p}_{h}^{m+1}\right)$ is the numerical solution of the finite-difference scheme $(2.7)-(2.8)$, and $(\mathbf{u}(\mathbf{x}, t), p(\mathbf{x}, t), \theta(\mathbf{x}, t))$ is the solution of the thermoporoelastic problem (1.1)-(1.3). If $\mathbf{u}_{h}^{0}, p_{h}^{0}, \theta_{h}^{0}$ are $\mathcal{O}\left(h^{2}\right)$ approximations of $\mathbf{u}(\mathbf{x}, 0), p(\mathbf{x}, 0)$ and $\theta(\mathbf{x}, 0)$ respectively, then

$$
\left\|\delta \mathbf{u}_{h}^{m+1}\right\|_{A} \leq C_{1}\left(h^{2}+\tau\right), \quad\left\|\delta \mathbf{p}_{h}^{m+1}\right\|_{C} \leq C_{2}\left(h^{2}+\tau\right),
$$

where $C_{1}$ and $C_{2}$ are constants independent of $h$ and $\tau$.

\section{Multigrid Solution Method}

We are interested to develop efficient iterative multigrid solution methods for solving the large system of equations corresponding to (2.7)-(2.8)

$$
\mathbf{L}_{h} \mathbf{v}_{h}=\mathbf{s}_{h}
$$

Traditional understanding of multigrid is based on the insight that a smoothing method reduces high frequency components of an error between the numerical approximation and the exact numerical solution, and a coarse grid correction based on standard grid coarsening handles the low frequency error components. The design of efficient relaxation methods for the multigrid solution of systems of partial differential equations often requires special attention. The smoother should smooth the error for all unknowns in the equations (that are possibly of different type) of a system. A multigrid method with a suitable relaxation method can be chosen on the basis of Fourier analysis. We will not focus on Fourier analysis in this paper. If the differential operator that corresponds to the primary unknown in each equation is the leading operator, smoothing is a straightforward matter, and a simple equation-wise decoupled relaxation method can be efficiently used. If, however, one of the operators on the system's main diagonal after discretization equals zero or is very close to zero, the choice of an efficient smoother needs care [19]. Notice that the coefficients in the main 
diagonal operators corresponding to the pressure and temperature equations of the system considered here, are typically very small. A first obvious choice in the case of strong off-diagonal operators in the differential system is coupled smoothing: All unknowns in the system at a certain grid point are updated simultaneously. Decoupled smoothing, however, is to be preferred, and it is typically found in the distributive framework: smoothing is applied after a post-conditioning step of the original system [6, 22]. For poroelastic problems, it was found in [10] that distributive smoothers are more robust than coupled relaxation, especially when very small time steps are involved. For this reason, this strategy is considered here.

\subsection{Distributive Smoothing}

An elegant way to describe distributive relaxation is to introduce a right preconditioner in the smoothing procedure [21]. This means that we introduce new variables $\mathbf{w}_{h}$, where $\mathbf{v}_{h}=\mathbf{C}_{h} \mathbf{w}_{h}$, and consider the transformed system $\mathbf{L}_{h} \mathbf{C}_{h} \mathbf{w}_{h}=\mathbf{s}_{h}$, with $\mathbf{C}_{h}$ chosen in such a way that the resulting operator $\mathbf{L}_{h} \mathbf{C}_{h}$ is suited for decoupled (non-collective) relaxation. The distributive relaxation can then be described in the following way:

- Transform the system $\mathbf{L}_{h} \mathbf{v}_{h}=\mathbf{s}_{h}$, to a simpler one by a suitable preconditioning with an operator $\mathbf{C}_{h}$ (the distributor).

- Choose a point- or line-wise relaxation process, preferably for each of the equations of the transformed system separately, of the form

$$
\mathbf{w}_{h}^{m+1}=\mathbf{w}_{h}^{m}+\mathbf{B}_{h}\left(\mathbf{s}_{h}-\mathbf{L}_{h} \mathbf{C}_{h} \mathbf{w}_{h}^{m}\right)
$$

with $\mathbf{B}_{h}$ being some approximation of the inverse of $\mathbf{L}_{h} \mathbf{C}_{h}$.

- Reformulate this relaxation scheme in terms of the original operator and unknowns by using $\mathbf{v}_{h}=\mathbf{C}_{h} \mathbf{w}_{h}$ :

$$
\mathbf{v}_{h}^{m+1}=\mathbf{v}_{h}^{m}+\mathbf{C}_{h} \mathbf{B}_{h}\left(\mathbf{s}_{h}-\mathbf{L}_{h} \mathbf{v}_{h}^{m}\right)
$$

In detail, the distributive relaxation consists of two steps, the predictor and the corrector. In the predictor step, a new approximation $\delta \mathbf{w}_{h}^{m+1}$ to the ghost variable $\delta \mathbf{w}_{h}$ is computed,

$$
\mathbf{L}_{h} \mathbf{C}_{h} \delta \mathbf{w}_{h}^{m+1}=\mathbf{r}_{h}^{m}
$$

with residual $\mathbf{r}_{h}^{m}=\mathbf{s}_{h}-\mathbf{L}_{h} \mathbf{v}_{h}^{m}$. In the corrector step, the new approximation for $\mathbf{v}_{h}^{m}$ is then added to the present approximation as

$$
\mathbf{v}_{h}^{m+1}=\mathbf{v}_{h}^{m}+\mathbf{C}_{h} \delta \mathbf{w}_{h}^{m+1} .
$$

Regarding the fully discrete thermoporoelastic problem (2.7)-(2.8), we have to solve on each time step, the problem $\mathbf{L}_{h} \mathbf{v}_{h}^{k+1}=\mathbf{s}_{h}^{k+1}$, where

$$
\mathbf{L}_{h}=\left(\begin{array}{cc}
A & \widetilde{G} \\
\widetilde{D} & C+\tau B
\end{array}\right), \quad \mathbf{v}_{h}^{k+1}=\left(\begin{array}{c}
\mathbf{u}_{h}^{k+1} \\
\mathbf{p}_{h}^{k+1}
\end{array}\right), \quad \mathbf{s}_{h}^{k+1}=\left(\begin{array}{c}
\mathbf{g}_{h}^{k+1} \\
\tau \mathbf{f}_{h}^{k+1}+\widetilde{D} \mathbf{u}_{h}^{k}+C \mathbf{p}_{h}^{k}
\end{array}\right) .
$$


The distributive smoothing procedure we propose here for discrete problem (4.1) is defined in the following way: First, we introduce the help variables $\overline{\mathbf{w}}_{h}=\left(\mathbf{w}_{h}, \mathbf{q}_{h}\right)^{t}$ as

$$
\left(\begin{array}{c}
\mathbf{u}_{h} \\
\mathbf{p}_{h}
\end{array}\right)=\mathbf{C}_{h}\left(\begin{array}{l}
\mathbf{w}_{h} \\
\mathbf{q}_{h}
\end{array}\right)=\left(\begin{array}{cc}
I_{h} & -\widetilde{G} \\
\mathbf{C}_{h}^{(2,1)} & \mathbf{C}_{h}^{(2,2)}
\end{array}\right)\left(\begin{array}{l}
\mathbf{w}_{h} \\
\mathbf{q}_{h}
\end{array}\right)
$$

where $\mathbf{C}_{h}^{(2,1)} \mathbf{w}_{h}=\frac{\lambda+\mu}{2}\left(\frac{1}{\alpha_{p}} D \mathbf{w}_{h}, \frac{1}{\alpha_{\theta}} D \mathbf{w}_{h}\right)^{t}$ and operator $\mathbf{C}_{h}^{(2,2)}$ is defined as

$$
\mathbf{C}_{h}^{(2,2)}=\left(\begin{array}{cc}
-\frac{\lambda+2 \mu}{\alpha_{p}} \Delta_{h} & 0 \\
0 & -\frac{\lambda+2 \mu}{\alpha_{\theta}} \Delta_{h}
\end{array}\right) .
$$

The transformed operator $\mathbf{L}_{h} \mathbf{C}_{h}$, then reads

$$
\mathbf{L}_{h} \mathbf{C}_{h}=\left(\begin{array}{cc}
\mu \Delta_{h}^{\mathbf{u}} & 0 \\
\mathbf{L} \mathbf{C}_{h}^{(2,1)} & \mathbf{L C}_{h}^{(2,2)}
\end{array}\right)
$$

with $\Delta_{h}^{\mathbf{u}}=\operatorname{diag}\left(\Delta_{h}^{u}, \Delta_{h}^{v}\right)$ and

$$
\begin{aligned}
\mathbf{L C}_{h}^{(2,2)}= & \left(\begin{array}{cc}
-\left(\alpha_{p}+(\lambda+2 \mu) c_{11} / \alpha_{p}\right) \Delta_{h} & -\left(\alpha_{p}+(\lambda+2 \mu) c_{12} / \alpha_{\theta}\right) \Delta_{h} \\
-\left(\alpha_{\theta}+(\lambda+2 \mu) c_{12} / \alpha_{p}\right) \Delta_{h} & -\left(\alpha_{\theta}+(\lambda+2 \mu) c_{22} / \alpha_{\theta}\right) \Delta_{h}
\end{array}\right) \\
& +(\lambda+2 \mu) \tau\left(\begin{array}{cc}
\frac{k_{p}}{\alpha_{p}} \Delta_{h}^{2} & 0 \\
0 & \frac{k_{\theta}}{\alpha_{\theta}} \Delta_{h}^{2}
\end{array}\right) .
\end{aligned}
$$

This transformed operator is triangular, so it is suited for decoupled smoothing. The first two equations in (4.2) can be smoothed with an efficient relaxation process for the Laplace operator. This is typically the well-known red-black Gauss-Seidel relaxation (in 2D and 3D) [19], which is well parallelizable. The corresponding smoothing factor in $2 \mathrm{D}$ is 0.063 for two iterations.

A challenging task here is to find a highly efficient smoother for the last two equations in (4.2),

$$
\mathbf{L C}_{h}^{(2,2)} \delta \mathbf{q}_{h}=\widetilde{\mathbf{r}}_{h},
$$

which corresponds to a coupled system where Laplace and biharmonic operators occur. The chosen approach here, is to split the operator as follows

$$
-\widetilde{\Delta}_{h} \mathbf{z}_{h}=\widetilde{\mathbf{r}}_{h}, \quad \mathbf{R}_{h} \delta \mathbf{q}_{h}=\mathbf{z}_{h}
$$

where $\widetilde{\Delta}_{h}=\operatorname{diag}\left(\Delta_{h}, \Delta_{h}\right)$ and operator $\mathbf{R}_{h}$ correspond to a coupled reactiondiffusion problem, where

$$
\begin{aligned}
\mathbf{R}_{h}= & \left(\begin{array}{cc}
\alpha_{p}+(\lambda+2 \mu) c_{11} / \alpha_{p} & \alpha_{p}+(\lambda+2 \mu) c_{12} / \alpha_{\theta} \\
\alpha_{\theta}+(\lambda+2 \mu) c_{12} / \alpha_{p} & \alpha_{\theta}+(\lambda+2 \mu) c_{22} / \alpha_{\theta}
\end{array}\right) \\
& -(\lambda+2 \mu) \tau\left(\begin{array}{cc}
\frac{k_{p}}{\alpha_{p}} \Delta_{h} & 0 \\
0 & \frac{k_{\theta}}{\alpha_{\theta}} \Delta_{h}
\end{array}\right) .
\end{aligned}
$$


with an extra variable $\mathbf{z}_{h}$. The first problem in (4.3) corresponds to two simple Laplace problems which can be smoothed again with red-black Gauss-Seidel iteration. For the second problem in (4.3), the coupled reaction-diffusion equation, we consider a collective red-black Gauss-Seidel with a relaxation parameter $\omega=0.75$. This relaxation parameter has been chosen on the basis of computing experiments. The distributive relaxation is designed such that its performance should be independent of problem parameters, like the Lamé coefficients or the time step. This is confirmed in the section devoted to numerical experiments.

\subsection{Coarse grid correction}

In a multigrid method for staggered grid discretizations one chooses standard geometric grid coarsening, i.e., the sequence of coarse grids is obtained by doubling the mesh size in each space direction. An appropriate coarse grid correction consists of straightforward geometric transfer operators $R_{h, 2 h}, P_{2 h, h}$, which are well-established in the field of computational fluid dynamics and direct coarse grid discretizations. The transfer operators that act on the different unknowns are dictated by the staggered grid, therefore we have to distinguish the transfer operators which act on the different grids $\omega_{u}, \omega_{v}$ and $\omega_{p}$. For the components of the displacements we consider 6-point restrictions and for scalar fields a 9-point restriction. In stencil notation they are given by

$$
\begin{aligned}
& R_{h, 2 h}^{u}=\frac{1}{8}\left[\begin{array}{lll}
1 & & 1 \\
2 & \star & 2 \\
1 & & 1
\end{array}\right], \quad R_{h, 2 h}^{v}=\frac{1}{8}\left[\begin{array}{lll}
1 & 2 & 1 \\
& \star & \\
1 & 2 & 1
\end{array}\right], \\
& R_{h, 2 h}^{p}=R_{h, 2 h}^{\theta}=\frac{1}{16}\left[\begin{array}{lll}
1 & 2 & 1 \\
2 & 4 & 2 \\
1 & 2 & 1
\end{array}\right],
\end{aligned}
$$

respectively. The restriction operator for scalar fields differs from the usual one in solving the incompressible Navier-Stokes equations, because of the placement of pressure points at the vertices, whereas a cell-centered pressure grid is employed in fluid mechanics applications. As the prolongation operators $P_{2 h, h}^{u}$, $P_{2 h, h}^{v}, P_{2 h, h}^{p}$ and $P_{2 h, h}^{\theta}$, one applies the usual interpolation operators based on bilinear interpolation of neighboring coarse grid unknowns, dictated by the staggered grid.

\section{$5 \quad$ Numerical Experiments}

\subsection{One-dimensional column problem}

In the first numerical experiment we show the unstable behaviour of standard finite-difference schemes on collocated grids, in order to motivate the use of staggered grids. We consider an idealized problem consisting a onedimensional column of fluid-saturated porous media occupying the region $0<x<H=20$, initially being at a temperature of $\theta=0^{\circ} C$. The column is bounded by rigid, impermeable bottom and walls. The fluid flows freely through the top surface 
Table 1. Material parameters for the one-dimensional column problem.

\begin{tabular}{llll}
\hline Parameter & Definition & Value & Unit \\
\hline$\alpha_{p}$ & Biot Willis constant & 0.95 & - \\
$\alpha_{\theta}$ & Thermal expansion coefficient & $0.125^{*} \alpha_{p}$ & - \\
$\kappa_{p}$ & Hydraulic conductivity & 0.534 & $\mathrm{~m}^{2} / \mathrm{MPa}$ day \\
$\kappa_{\theta}$ & Thermal conductivity & $4^{*} \kappa_{p}$ & $\mathrm{Wm}-1 \mathrm{C}^{-1}$ \\
$M$ & Biot modulus & $10^{4}$ & $\mathrm{~kg} / \mathrm{ms}$ \\
$C_{M}$ & Volumetric heat capacity & $10^{-3}$ & $\mathrm{~J} /\left(\mathrm{m}^{3} \mathrm{C}\right)$ \\
$\beta_{M}^{\prime}$ & Thermal expansion coefficient & $10^{-5}$ & ${ }^{\circ} \mathrm{C}^{-1}$ \\
\hline
\end{tabular}

at atmospheric pressure and therefore a null pressure is imposed. A uniform load of intensity of $\sigma_{0}=-100 \mathrm{~N} / \mathrm{m}^{2}$ is applied on the top of the column. The temperature in the boundary is kept constant, assuming that the walls of the column are isolated. The considered material properties are indicated in Table 1. This problem is an extension of the classical onedimensional problem of Terzaghi. Under these conditions, we have to solve the following onedimensional version of $(1.1)-(1.3)$

$$
\begin{aligned}
& -\frac{\partial^{2} u}{\partial x^{2}}+\alpha_{p} \frac{\partial p}{\partial x}+\alpha_{\theta} \frac{\partial \theta}{\partial x}=0 \\
& \frac{1}{M} \frac{\partial p}{\partial t}-3 \beta_{M}^{\prime} \frac{\partial \theta}{\partial t}+\alpha_{p} \frac{\partial}{\partial t}\left(\frac{\partial u}{\partial x}\right)-\kappa_{p} \frac{\partial^{2} p}{\partial x^{2}}=0 \\
& -3 \beta_{M}^{\prime} \frac{\partial p}{\partial t}+c_{M} \frac{\partial \theta}{\partial t}+\alpha_{\theta} \frac{\partial}{\partial t}\left(\frac{\partial u}{\partial x}\right)-\kappa_{\theta} \frac{\partial^{2} \theta}{\partial x^{2}}=0
\end{aligned}
$$

$x \in(0, H)$, subject to the boundary conditions

$$
\begin{aligned}
& \frac{\partial u}{\partial x}(0, t)=\sigma_{0}, \quad p(0, t)=\theta(0, t)=0, \quad t \in(0, T] \\
& u(H, t)=0, \quad \frac{\partial p}{\partial x}(H, t)=0, \quad \theta(H, t)=0, \quad t \in(0, T]
\end{aligned}
$$

and the initial conditions

$$
\frac{\partial u}{\partial x}(x, 0)=0, \quad p(x, 0)=\theta(x, 0)=0, \quad x \in(0, H) .
$$

If a collocated mesh in space is used and the first derivative terms are discretized by central differences, strong nonphysical oscillations can appear in the approximation of the pressure and temperature fields in the early time of the consolidation process. Figures 1 (a) and 1(b) show for $T=10^{-4}$ that a spatial discretization by central differences on a collocated mesh composed by 65 nodes combined with one step of a backward time discretization, leads to spurious oscillations in the approximation of the pressure and the temperature. This instability of the numerical scheme can be avoided if the chosen space discretization parameter $\mathrm{h}$ is small enough. However, for multidimensional problems, this kind of restriction can require many nodes and therefore an excessive computational effort. So, the use of staggered meshes, which generate 
solutions free of oscillations independently of the discretization parameters, is totally justified. In Figures 1(a) and 1(b), it is observed that the spurious oscillations for both pressure and temperature, disappear when a staggered mesh in space is used.

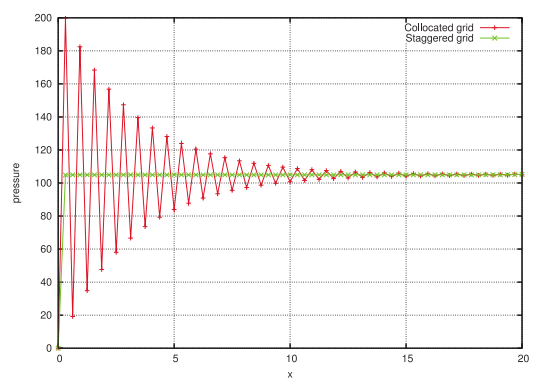

(a)

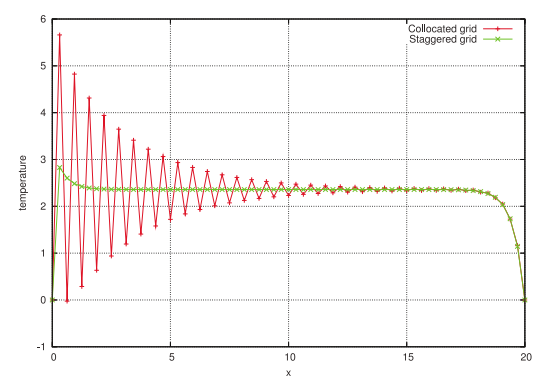

(b)

Figure 1. Approximation solutions for pressure (a) and for temperature (b) using collocated and staggered grids for the column problem.

\subsection{Analytical solution of a 2D model problem}

We consider a 2D problem defined on the unit square with Dirichlet boundary conditions. The source terms and the boundary and initial conditions are such that the analytical solution is given by

$$
\begin{array}{ll}
u(x, y, t)=\cos (\pi x) \sin (\pi y) \exp (-\pi t), & v(x, y, t)=\sin (\pi x) \cos (\pi y) \exp (-\pi t), \\
p(x, y, t)=\sin (\pi x) \sin (\pi y) \exp (-\pi t), & \theta(x, y, t)=\sin (\pi x) \sin (\pi y) \exp (-\pi t),
\end{array}
$$

To approximate this problem we use scheme $(2.7)-(2.8)$ on a staggered grid in space as described in Section 2.1. In the numerical experiments the parameter settings chosen are $\alpha_{p}=0.9, \alpha_{\theta}=0.8, \kappa_{p}=10^{-4}, \kappa_{\theta}=10^{-5}, M=10^{7}$, $c_{M}=10^{-5}, \beta_{M}^{\prime}=10^{-10}$. The Young's modulus and the Poisson's ratio, characterizing the elastic solid, are $E=3 \times 10^{4}$ and $\nu=0.2$ respectively. The Lamé coefficients $\lambda$ and $\mu$ are related to the Young's modulus $E$ and the Poisson's ratio $\nu$ by

$$
\lambda=\frac{\nu E}{(1+\nu)(1-2 \nu)}, \quad \mu=\frac{E}{2(1+\nu)} .
$$

Table 2 presents the difference between analytic and numerical solution in energy norms for displacements, pressure and temperature, i.e., $\left\|\delta \mathbf{u}_{h}\right\|_{A}$ and $\left\|\delta \mathbf{p}_{h}\right\|_{C}$ at final time $T=10^{-2}$ and several values of discretization parameters $h$ and $\tau$. We also display the corresponding orders of convergence. As it can be observed, first order convergence is obtained, being these numerical results in agreement with Proposition 2.

At each time level the corresponding linear system is solved by multigrid with the distributive smoother proposed in this work. The measure for multigrid convergence is related to the absolute value of the residual after the $m t h$ 
Table 2. Energy norms of the errors and orders of convergence.

\begin{tabular}{lllll}
\hline & $h=1 / 32$ & $h=1 / 64$ & $h=1 / 128$ & $h=1 / 256$ \\
$\tau=1 / 40$ & $\tau=1 / 80$ & $\tau=1 / 160$ & $\tau=1 / 320$ \\
\hline$\left\|\delta \mathbf{u}_{h}\right\|_{A}$ & 3.1221 & 1.5602 & 0.7802 & 0.3902 \\
$\left\|\delta \mathbf{p}_{h}\right\|_{C}$ & 1.0008 & 0.9998 & 0.9996 & 0.2385 \\
& 1.7081 & 0.9107 & 0.4697 & \\
\hline
\end{tabular}

iteration in the maximum norm over the four equations in the system, that is,

$$
\operatorname{res}_{h}^{m}=\left|r_{1, h}^{m}\right|+\left|r_{2, h}^{m}\right|+\left|r_{3, h}^{m}\right|+\left|r_{4, h}^{m}\right|
$$

being $r_{i, h}^{m}$ the residual of the $i t h$ equation in the system. The multigrid convergence factor $\rho_{h}$ presented in the tables below is then given by

$$
\rho_{h}=\sqrt[5]{\operatorname{res}_{h}^{m} / \operatorname{res}_{h}^{m-5}}
$$

being $m$ the last iteration is chosen before the stopping criterion is met. This quantity is typically somewhat better than the asymptotic convergence factor. The stopping criterion is chosen as the absolute residual over all unknowns to be less than $10^{-9}$. This criterion is too severe for realistic applications, but well-suited for our investigation of the multigrid convergence. A matrixfree, stencil based version of multigrid is used. $\mathrm{F}(1,1)$, for example, denotes an F-cycle with one pre- and one post-relaxation. We only show results with F-cycle because V-cycle gives unsatisfactory results. We consider here the multigrid convergence in the first time step with different mesh sizes, ranging from $h=1 / 32$ to $h=1 / 256$ and for different time steps. These convergence statistics are representative for all other time steps. The time step is chosen very small: it ranges from $10^{-2}$ to $10^{-6}$. In the case of pressure or/and temperature boundary layers in the initial stage of a thermoporoelastic process, small time steps are realistic. Notice that for small time steps, the block diagonal of the corresponding system tends to zero, doing the system more difficult to solve.

Table 3. Convergence factors and number of iterations of the multigrid method.

\begin{tabular}{lllll}
\hline & $h=1 / 32$ & $h=1 / 64$ & $h=1 / 128$ & $h=1 / 256$ \\
\hline$\tau=10^{-2}$ & $0.124(17)$ & $0.141(18)$ & $0.147(19)$ & $0.148(20)$ \\
$\tau=10^{-4}$ & $0.093(16)$ & $0.092(16)$ & $0.115(17)$ & $0.135(18)$ \\
$\tau=10^{-6}$ & $0.081(16)$ & $0.082(16)$ & $0.095(16)$ & $0.107(17)$ \\
$\tau=10^{-8}$ & $0.080(16)$ & $0.081(16)$ & $0.081(16)$ & $0.082(16)$ \\
\hline
\end{tabular}

Table 3 shows the $\mathrm{F}(2,2)$-cycles results, presenting the multigrid convergence factor (5.3) and the number of iterations to reach the stopping criterion in brackets. An $h$-independent convergent can be observed in the table for the $\mathrm{F}(2,2)$-cycle and the distributive smoother considered in this work. Moreover, the multigrid convergence is independent of the time step. Even a better convergence is seen for extremely small time steps. 
Table 4. Multigrid convergence. Variation of the properties of the elastic solid.

\begin{tabular}{lllll}
\hline & $h=1 / 32$ & $h=1 / 64$ & $h=1 / 128$ & $h=1 / 256$ \\
\hline$\nu=0.25$ & $0.124(17)$ & $0.141(18)$ & $0.147(19)$ & $0.148(20)$ \\
$\nu=0.4$ & $0.140(18)$ & $0.145(18)$ & $0.148(20)$ & $0.148(21)$ \\
$\nu=0.45$ & $0.142(18)$ & $0.147(19)$ & $0.148(20)$ & $0.148(21)$ \\
$\nu=0.49$ & $0.146(20)$ & $0.148(21)$ & $0.148(22)$ & $0.148(23)$ \\
$\nu=0.499$ & $0.146(23)$ & $0.148(23)$ & $0.149(24)$ & $0.149(25)$ \\
\hline
\end{tabular}

We now vary the properties of the elastic solid and investigate their effect on the multigrid convergence. The time step for these tests is $\tau=10^{-2}$. Table 4 presents the $\mathrm{F}(2,2)$-cycle multigrid convergence in the first time step. In particular, the multigrid convergence (5.3) and the number of iterations to reduce the absolute value of the residual to less than $10^{-9}$ are shown. We fix the Young's modulus $E=3 \times 10^{4}$ and vary the Poisson's ratio $\nu$. A fast and robust multigrid convergence in the limit $\nu \rightarrow 0.5$ can be observed. Overall, all the results are impressive for such a complicated system.

\subsection{A footing problem}

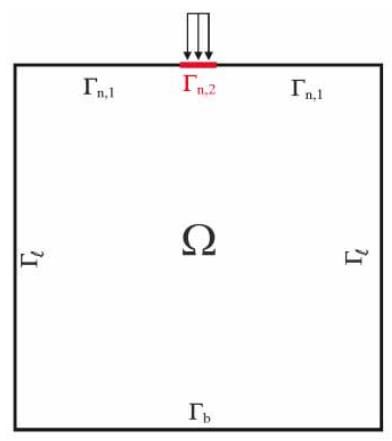

Figure 2. Computational domain for the footing problem.

The aim of this last experiment is to show the good performance of the proposed multigrid method for a realistic problem. It corresponds to a vertical cross-section through a homogeneous soil. The simulation domain is a 100 by 100 meters block, $\Omega=(-50,50) \times(0,100)$, as in Figure 2 . At the base of this domain the soil is assumed to be fixed, i.e. both horizontal and vertical components of displacements are taken as zero, while at some centered upper part of the domain a uniform load $\sigma_{0}=10^{3} \mathrm{~N} / \mathrm{m}^{2}$ is applied in a strip of length $40 \mathrm{~m}$ as depicted in Figure 2. The remaining of the top surface is assumed to be traction free. Horizontal displacement and vertical surface traction are assumed to be zero on each of the vertical walls. Concerning to the pressure, we prescribe the pore pressure at the top and lateral boundaries as zero, and we assume the bottom boundary to be impermeable. Temperature is kept constant 
in the whole domain. More precisely, the boundary data are given as follows.

$$
\begin{aligned}
& p=0, \quad \theta=0, \quad \sigma_{x y}=0, \quad \sigma_{y y}=0, \quad \text { on } \Gamma_{n, 1}, \\
& p=0, \quad \theta=0, \quad \sigma_{x y}=0, \quad \sigma_{y y}=-\sigma_{0}, \quad \text { on } \Gamma_{n, 2} \text {, } \\
& p=0, \quad \theta=0, \quad \sigma_{x y}=0, \quad u=0, \quad \text { on } \Gamma_{\ell}, \\
& p=0, \quad \theta=0, \quad v=0, \quad u=0, \quad \text { on } \Gamma_{b} \text {, }
\end{aligned}
$$

where $\sigma_{x y}=\mu\left(\frac{\partial u}{\partial y}+\frac{\partial v}{\partial x}\right), \sigma_{y y}=\lambda \frac{\partial u}{\partial x}+(\lambda+2 \mu) \frac{\partial v}{\partial y}$, and

$$
\begin{aligned}
& \Gamma_{n, 1}=\{(x, y) \in \partial \Omega, /|x|>20, y=100\} \\
& \Gamma_{n, 2}=\{(x, y) \in \partial \Omega, /|x| \leq 20, y=100\} \\
& \Gamma_{\ell}=\{(x, y) \in \partial \Omega, /|x|=50\}, \quad \Gamma_{b}=\{(x, y) \in \partial \Omega, / y=0\} .
\end{aligned}
$$

Table 5. Material parameters for the two dimensional footing problem.

\begin{tabular}{llll}
\hline Parameter & Definition & Value & Unit \\
\hline$E$ & Young's modulus & $3 \times 10^{4}$ & $\mathrm{~N} / \mathrm{m}^{2}$ \\
$\nu$ & Poisson's ratio & 0.2 & - \\
$\alpha_{p}$ & Biot Willis constant & 0.9 & - \\
$\alpha_{\theta}$ & Thermal expansion coefficient & 0.8 & - \\
$\kappa_{p}$ & Hydraulic conductivity & $10^{-9}$ & $\mathrm{~m}^{2} / \mathrm{MPa}$ day \\
$\kappa_{\theta}$ & Thermal conductivity & $10^{-11}$ & $\mathrm{Wm} \mathrm{m}^{-1} \mathrm{C}^{-1}$ \\
$M$ & Biot modulus & $10^{-7}$ & $\mathrm{~kg} / \mathrm{ms}^{-7}$ \\
$C_{M}$ & Volumetric heat capacity & $10^{-3}$ & $\mathrm{~J} /\left(\mathrm{m}^{3} \mathrm{C}\right)$ \\
$\beta_{M}^{\prime}$ & Thermal expansion coefficient & $10^{-10}$ & ${ }^{\circ} \mathrm{C}^{-1}$ \\
\hline
\end{tabular}

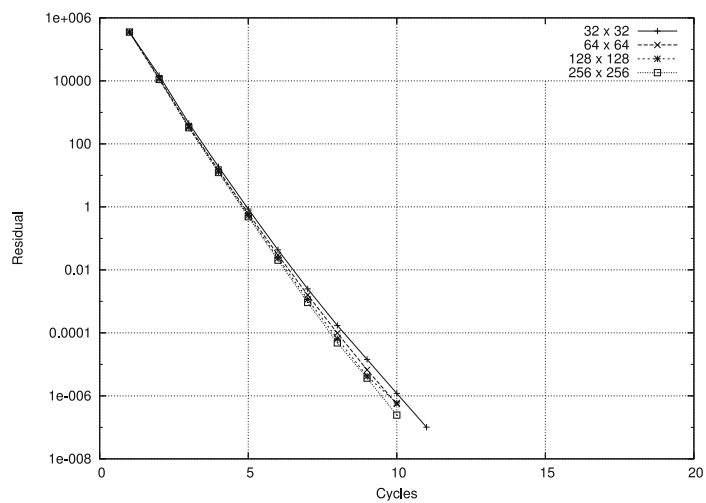

Figure 3. Multigrid convergence $\mathrm{F}(2,2)$-cycle, pointwise distributive smoother.

The material properties of the porous medium for this case are given in Table 5. In this experiment we choose the time discretization parameter as $\tau=10^{-1}$. No oscillations have been observed for both scalar solution fields, pressure and temperature. An F(2,2)-cycle is applied each time step with the 
pointwise distributive smoother. The stopping criterion per time step was chosen here as the absolute residual to be less than $10^{-6}$.

The multigrid convergence during the first time step, for different numbers of spatial mesh points $32^{2}, 64^{2}, 128^{2}$ and $256^{2}$ is presented in Figure 3 . We again confirm the robustness and efficiency of the proposed multigrid method and in particular of the considered smoother.

\section{References}

[1] A. Belotserkovets and J.H. Prevost. Thermoporoelastic response of a fluidsaturated porous sphere: An analytical solution. Internat. J. Engrg. Sci., 49:1415-1423, 2011. http://dx.doi.org/10.1016/j.ijengsci.2011.05.017.

[2] M. Biot. General theory of three dimensional consolidation. J. Appl. Phys., 12:155-169, 1941. http://dx.doi.org/10.1063/1.1712886.

[3] M. Biot. General solutions of the equations of elasticity and consolidation of a porous material. J. Appl. Phys., 23:91-96, 1956.

[4] N. Boal, F.J. Gaspar, F.J. Lisbona and P.N. Vabishchevich. Finite difference analysis of fully dynamic problems for saturated porous media. J. Comput. Appl. Math., 236:1090-1102, 2011. http://dx.doi.org/10.1016/j.cam.2011.07.032.

[5] N. Boal, F.J. Gaspar, F.J. Lisbona and P.N. Vabishchevich. Finite difference analysis of a double-porosity consolidation model. Numer. Methods Partial Differential Equations, 28:138-154, 2012. http://dx.doi.org/10.1002/num.20612.

[6] A. Brandt and N. Dinar. Multigrid solutions to elliptic flow problems. In S. Parter(Ed.), Numer. Methods Partial Differential Equations, pp. 53-147, New York, 1979. Academic Press.

[7] F. Brezzi. On the existence, uniqueness and approximation of saddle-point problems arising from Lagrange multipliers. R.A.I.R.O, 8:129-151, 1974.

[8] O. Coussy. Mechanics of Porous Continua. John Wiley, 1995.

[9] F.J. Gaspar, J.L. Gracia, F.J. Lisbona and P.N. Vabishchevich. A stabilized method for a secondary consolidation Biot's model. Numer. Methods Partial Differential Equations, 24:60-78, 2008. http://dx.doi.org/10.1002/num.20242.

[10] F.J. Gaspar, F.J. Lisbona, C.W. Oosterlee and R. Wienands. A systematic comparison of coupled and distributive smoothing in multigrid for the poroelasticity system. Numer. Linear Algebra Appl., 11:93-113, 2004. http://dx.doi.org/10.1002/nla.372.

[11] F.J. Gaspar, F.J. Lisbona and P.N. Vabishchevich. A finite difference analysis of Biot's consolidation model. Appl. Numer. Math., 44:487-506, 2003. http://dx.doi.org/10.1016/S0168-9274(02)00190-3.

[12] F.J. Gaspar, F.J. Lisbona and P.N. Vabishchevich. Staggered grid discretizations for the quasi-static Biot's consolidation problem. Appl. Numer. Math., 56:888898, 2006. http://dx.doi.org/10.1016/j.apnum.2005.07.002.

[13] R.L. Lewis and B.A. Schrefler. The Finite Element Method in the Static and Dynamic Deformation and Consolidation of Porous Media. Wiley Chichester, 1998.

[14] D.F. McTigue. Thermoelastic response of fluid-saturated porous rock. J. Geophys. Res., 91(B9):9533-9542, 1986.

http://dx.doi.org/10.1029/JB091iB09p09533. 
[15] M.A. Murad and A.F.D. Loula. Improved accuracy in finite element analysis of Biot's consolidation problem. Comput. Methods Appl. Mech. Engrg., 95:359-382, 1992. http://dx.doi.org/10.1016/0045-7825(92)90193-N.

[16] A.A. Samarskii. The Theory of Difference Schemes. Marcel Dekker Inc., New York, Basel, 2001.

[17] D. Shin and J. Strikwerda. Inf-Sup conditions for finite-difference approximations of the Stokes equations. J. Austral. Math. Soc. Ser., 39:121-134, 1997. http://dx.doi.org/10.1017/S0334270000009255.

[18] K. Terzaghi. Theoretical Soil Mechanics. Wiley, New York, 1942.

[19] U. Trottenberg, C.W. Oosterlee and A. Schüller. Multigrid. Academic Press, New York, 2001.

[20] R. Wienands, F.J. Gaspar, F.J. Lisbona and C.W. Oosterlee. An efficient multigrid solver based on distributive smoothing for poroelasticity equations. Computing, 73:99-119, 2004. http://dx.doi.org/10.1007/s00607-004-0078-y.

[21] G. Wittum. Multi-grid methods for Stokes and Navier-Stokes equations with transforming smoothers: algorithms and numerical results. Numer. Math., 54:543-563, 1989. http://dx.doi.org/10.1007/BF01396361.

[22] G. Wittum. On the convergence of multi-grid methods with transforming smoothers. Numer. Math., 57:15-38, 1990.

http://dx.doi.org/10.1007/BF01386394. 